\title{
ESPRIT with multiple-angle subarray beamforming
}

\author{
Wen Xu, Ying Jiang ${ }^{*}$ and Huiquan Zhang
}

\begin{abstract}
This article presents a new approach of implementing signal direction-of-arrival estimation, in which subarray beamforming is applied prior to estimation of signal parameters via rotational invariance techniques (ESPRIT). Different from the previous approaches, the beam-domain data from multiple adjacent pointing angles are combined in a way that the displacement invariance structure required by ESPRIT is maintained. It is intended to further obtain a sub-beamwidth resolution for a conventional multi-beam system already having small beamwidths. Computer simulations show that for typical multi-beam system applications the new approach provides improved estimation mean-square errors over the original ESPRIT, on top of reduced requirements for signal-to-noise ratio, number of snapshots, and computational time.
\end{abstract}

Keywords: Multi-beam system, Subarray beamforming, Direction-of-arrival estimation

\section{Introduction}

Multi-beam systems are commonly used in various sonar and radar applications. For example, multi-beam echo sounder has been a major instrument for mapping the seafloor. It uses a so-called Mills Cross array configuration, installed underneath a survey ship, transmitting a beam wide perpendicular to the ship's moving direction (across-track) and narrow parallel to the ship's moving direction (along-track) and forming multiple receiving beams narrow across-track and relatively wide along-track [1]. Hence, a strip of bottom points' acrosstrack is obtained from one transmission. One major problem with such a system is that the footprint spreads wider as the beam is steered away from the broadside direction; this, added with weakened bottom scattering strength at small grazing angles, causes serious degradation in resolution.

In this article, we develop a new approach to obtain sub-beamwidth resolution by applying high-resolution signal direction-of-arrival (DOA) estimation in beam domain. For the purpose of practical implementation, we choose estimation of signal parameters via rotational invariance techniques (ESPRIT) [2] as the method to be applied. ESPRIT is well known for its high resolution, computational efficiency, and robustness to array

\footnotetext{
* Correspondence: jiangying433@163.com

Department of Information Science and Electronic Engineering, Zhejiang University, Zheda Road \#38, Hangzhou 310027, China
}

imperfections. However, direct application of the ESPRIT to a multi-beam system can still be quite challenging if the number of array elements involved is large. Besides, the estimation performance is often limited by the available signal-to-noise ratio (SNR) and number of independent snapshots.

To achieve a reduction of computation, a so-called beamspace approach has been developed, which first projects the original data into a subspace of lower dimension (i.e., the beamspace) via non-adaptive beamforming and then processes the subspace data using high-resolution algorithms [3-6]. Because, the dimension of the beamspace is usually much smaller than that of the element-space, the computational load is reduced and the statistical stability in DOA estimation is improved. Besides, the beamspace approach can suppress interfering signals and noises outside the selected beams, thus increasing the effective SNR for highresolution processing.

One of the disadvantages with beamspace processing is that the performance can be degraded if the beam transformation is not properly done [4]. In the context of multi-beam applications previously mentioned, since the beamwidth is already small, the DOA estimation performance can be poorer when the source direction is around the edge of an effective beam. Our method in this article forms multiple adjacent beams closelyspaced and combines them in a way that the displacement invariance structure required by ESPRIT is maintained,

\section{穴}

(c) 2012 Xu et al.; licensee Springer. This is an Open Access article distributed under the terms of the Creative Commons Attribution License (http://creativecommons.org/licenses/by/2.0), which permits unrestricted use, distribution, and reproduction in any medium, provided the original work is properly cited. 
thus improving robustness to scalloping loss in beamforming. Moreover, for the same number of subarrays implementing beamforming, the number of resolvable source directions can be more.

The approach here is similar to several later generalizations of the original ESPRIT [7-12], but some obvious differences exist. For example, the multiinvariance ESPRIT, which applies the original ESPRIT in parallel to each multiple pair of translated subarrays of different displacements, is mostly done on the element space [7-9]; the new algorithm presented in Section "Case 2: $L>2$ ", on the other hand, applies the original ESPRIT to each multiple pair of translated beam outputs of different pointing angles. The same concept can also be seen in circular uniform beam arrays (CUBA)-ESPRIT [10] and reactance-domain (RD)-ESPRIT [11,12]. CUBA-ESPRIT employs a virtual transformation in beam pattern to apply the original ESPRIT to a CUBA antenna. RD-ESPRIT forms a correlation matrix from electronically steerable parasitic array radiators antenna's single output, by oversampling the output or transmitting the same signal in sequence, to allow the use of ESPRIT technique. Both methods employ beamforming on the entire array, while our approach is used on subarray and more flexible with varied combinations of different subarray configurations and different beampointing angles.

The rest of the article is organized as follows. In the following section, we describe the DOA estimation problem and the original ESPRIT algorithm. We then introduce in Section "ESPRIT applied to multiple-angle subarray beams" the approach of combining subarray beams of multiple adjacent point angles prior to ESPRIT processing; a proof of invariance structure is also provided. Section "Simulations" presents some simulation results in comparison with the conventional ESPRIT as well as the Cramer-Rao bound, and finally the conclusionis given at the end of the article.

\section{Problem statement}

Assume that there are $K$ uncorrelated far-field narrowband sources impinging on a linear array of $M$ equally spaced sensors with sensor spacing $d$ less than or equal to half wavelength. The $M \times 1$ received signal vector can be expressed as

$$
\mathbf{x}(t)=\mathbf{A s}(t)+\mathbf{n}(t)
$$

where $\mathbf{s}(t)=\left[\begin{array}{lll}s_{1}(t) & \cdots & s_{K}(t)\end{array}\right]^{T}, s_{k}(t)$ is the incident signal from the $k$ th source, $(\cdot)^{T}$ denotes the transpose, $\mathbf{A}=\left[\begin{array}{lll}\mathbf{a}\left(\theta_{1}\right) & \cdots & \mathbf{a}\left(\theta_{K}\right)\end{array}\right], \mathbf{a}\left(\theta_{k}\right)$ is the array manifold vector, $\theta_{k}$ denotes the signal arriving direction of the $k$ th source, and $\mathbf{n}(t)$ denotes the noise vector. For plane wave propagation, we have

$$
\mathbf{a}\left(\theta_{k}\right)=\left[1, e^{j 2 \pi d \sin \theta_{k} / \lambda}, \cdots, e^{j 2 \pi(M-1) d \sin \theta_{k} / \lambda}\right]^{T}
$$

where $\lambda$ is the wavelength. Suppose that the noise is zero-mean white Gaussian with variance $\sigma_{n}^{2}$ and uncorrelated with the source signals; the covariance of $\mathbf{x}(t)$ is then

$$
\mathbf{R}_{\mathbf{x x}}=\mathbf{A} \mathbf{R}_{\mathbf{s s}} \mathbf{A}^{H}+\sigma_{n}^{2} \mathbf{I}_{M}
$$

where $\mathbf{I}_{M}$ denotes an $M \times M$ identity matrix, $(\cdot)^{H}$ denotes the complex conjugate transpose, and $\mathbf{R}_{\mathbf{s s}}$ is the source covariance matrix of rank $K$.

The problem is to estimate source directions from $\mathbf{x}(t)$. Using ESPRIT, the first step is to form two identical subarrays separated by a common displacement $d_{s}$. Denote the number of sensors in the subarray by $M_{s}$ (we require $M_{s} \geq K+1$ ). The first subarray contains sensor 1 to $M_{s}$, while the second subarray contains sensor $M-$ $M_{s}+1$ to $M$. Obviously, the array manifold matrices associated with these two subarrays are related by $\mathbf{A}_{1} \boldsymbol{\Phi}=\mathbf{A}_{2}$, where $\boldsymbol{\Phi}$ is a $K \times K$ diagonal matrix with its diagonal element defined by $\phi_{k}=e^{j 2 \pi d_{s} \sin \theta_{k} / \lambda}$. Outputs from these two subarrays can then be written as

$$
\begin{aligned}
& \mathbf{x}_{1}(t)=\mathbf{A}_{1} \mathbf{s}(t)+\mathbf{n}_{1}(t) \\
& \mathbf{x}_{2}(t)=\mathbf{A}_{1} \mathbf{\Phi} \mathbf{s}(t)+\mathbf{n}_{2}(t)
\end{aligned}
$$

This re-structuring warrants that the signal sub-spaces associated with these two subarrays are related by a matrix solely determined by $\theta_{k}, k=1, \ldots, K$, which is then exploited to find the estimates of $\theta_{k}, k=$ $1, \ldots, K$. A commonly used version is the so-called TLS-ESPRIT, whose implementation can be summarized as follows [5]:

1. Obtain the sample covariance $\hat{R}_{x x}$, an estimate of $R_{x x}$.

2. Compute the eigendecomposition of $\hat{R}_{x x}$

$$
\hat{\mathbf{R}}_{\mathbf{x x}}=\hat{\mathbf{E}} \hat{\Lambda} \hat{\mathbf{E}}^{H}
$$

3. If necessary, estimate the number of sources, $K$.

4. Partition $\hat{\mathbf{E}}=\left[\hat{\mathbf{E}}_{\mathbf{s}} \mid \hat{\mathbf{E}}_{\mathbf{n}}\right]$, where $\hat{\mathbf{E}}_{\mathbf{s}}$ are the principal eigenvectors corresponding to the $K$ largest eigenvalues.

5. Compute the eigendecomposition of the $2 K \times 2 K$ matrix

$$
\left[\begin{array}{c}
\hat{\mathbf{E}}_{x}^{H} \\
\hat{\mathbf{E}}_{y}^{H}
\end{array}\right]\left[\begin{array}{ll}
\hat{\mathbf{E}}_{x} & \hat{\mathbf{E}}_{y}
\end{array}\right]=\mathbf{E} \boldsymbol{\Lambda} \mathbf{E}^{H}
$$

where $\hat{\mathbf{E}}_{x}$ and $\hat{\mathbf{E}}_{y}$, respectively, pick the first and the last $M_{\mathrm{s}}$ rows of $\hat{\mathbf{E}}_{\mathrm{s}}$. 
6. Partition $E$ into $K \times K$ submatrices

$$
\mathbf{E}=\left[\begin{array}{ll}
\mathbf{E}_{11} & \mathbf{E}_{12} \\
\mathbf{E}_{21} & \mathbf{E}_{22}
\end{array}\right]
$$

7. Calculate the eigenvalues $\gamma_{k}$ of $-\mathrm{E}_{12} \mathrm{E}_{22}^{-1}$, and then

$$
\hat{\theta}_{k}=\sin ^{-1}\left(-\frac{\lambda}{2 \pi d_{s}} \cdot \arg \gamma_{k}\right)
$$

where $\arg (\cdot)$ retrieves the angle information.

Estimation of the covariance requires sufficient statistical samples of both signals and noises, which is particularly demanding when the number of sensors is large. Besides, the computational complexity is in connection with the number of sensors as well.

\section{ESPRIT applied to multiple-angle subarray beams}

In this section, we present the algorithm of ESPRIT with subarray beamforming. The approach is similar to that in [4]; however, instead of using one output beam from each subarray, multiple output beams pointing to a group of closelyspaced angles are combined for ESPRIT processing.

We first divide the entire array into $N$ identical subarrays, each with $P$ sensors and equally separated by $\Delta,{ }^{\text {a }}$ which is an integer multiple of sensor spacing. The above parameters are related by $N=(M-P) / \Delta+1$. The received signal at each subarray can be expressed as

$$
\begin{gathered}
\mathbf{x}_{1}(t)=\mathbf{A}_{1} \mathbf{s}(t)+\mathbf{n}_{1}(t) \\
\mathbf{x}_{2}(t)=\mathbf{A}_{1} \boldsymbol{\Psi}_{1} \mathbf{s}(t)+\mathbf{n}_{2}(t) \\
\vdots \\
\mathbf{x}_{N}(t)=\mathbf{A}_{1} \boldsymbol{\Psi}_{N-1} \mathbf{s}(t)+\mathbf{n}_{N}(t)
\end{gathered}
$$

where $\mathbf{A}_{1}$ again denotes the array manifold for subarray $1, \quad \Psi_{1}$ is a $K \times K$ diagonal matrix with its diagonal element defined by $\psi_{k}=e^{j 2 \pi \Delta \sin \theta_{k} / \lambda}$, and $\Psi_{n}=$ $\left(\Psi_{1}\right)^{n}, n=1, \ldots, N-1$.

Denote the set of $L$ beam pointing angles as $\bar{\theta}_{l}, l=$ $1, \ldots, L$. The conventional beamforming weight matrix for each subarray is then

$$
\mathbf{W}_{b}=\left[a_{b}\left(\bar{\theta}_{1}\right) a_{b}\left(\bar{\theta}_{2}\right) \cdots a_{b}\left(\bar{\theta}_{L}\right)\right]
$$

where

$$
\mathbf{a}_{b}\left(\bar{\theta}_{l}\right)=\left[1, e^{\frac{j 2 \pi d \sin \bar{\theta}_{l}}{\lambda}}, \ldots, e^{j 2 \pi(P-1) d \sin \bar{\theta}_{l} / \lambda}\right]^{T}
$$

Note that the above weight matrix can be adjusted to control the mainlobe width and sidelobe levels, and in practical applications it should be calibrated for each beam pointing angles.
Case 1: $L=1$

We first look at the case with $L=1$, which was studied in [4]. In this case, $\mathbf{W}_{b}=a_{b}\left(\bar{\theta}_{1}\right)$, and the beamformer output at each subarray is

$$
\begin{gathered}
y_{1}(t)=a_{b}^{H}\left(\bar{\theta}_{1}\right) A_{1} s(t)+n^{\prime}{ }_{1}(t) \\
y_{2}(t)=a_{b}^{H}\left(\bar{\theta}_{1}\right) A_{1} \Psi_{1} s(t)+n_{2}^{\prime}(t) \\
\vdots \\
y_{N}(t)=a_{b}^{H}\left(\bar{\theta}_{1}\right) A_{1} \Psi_{N-1} s(t)+n_{N}^{\prime}(t)
\end{gathered}
$$

where $n_{i}^{\prime}(t)=a_{b}^{H}\left(\bar{\theta}_{1}\right) \mathbf{n}_{i}(t), i=1, \ldots, N$.

Treating each beam output as a virtual sensor, we now have a virtual array with $N$ equally spaced sensors. We further divide it into two subarrays of size $N-1$, whose data can be expressed as

$$
\begin{gathered}
\mathbf{z}_{1}(t)=\left[\begin{array}{c}
a_{b}^{H}\left(\bar{\theta}_{1}\right) \mathbf{A}_{1} \\
a_{b}^{H}\left(\bar{\theta}_{1}\right) \mathbf{A}_{1} \boldsymbol{\Psi}_{1} \\
\vdots \\
a_{b}^{H}\left(\bar{\theta}_{1}\right) \mathbf{A}_{1} \boldsymbol{\Psi}_{N-2}
\end{array}\right] s(t)+n^{\prime \prime}{ }_{1}(t) \\
\mathbf{z}_{2}(t)=\left[\begin{array}{c}
a_{b}^{H}\left(\bar{\theta}_{1}\right) \mathbf{A}_{1} \\
a_{b}^{H}\left(\bar{\theta}_{1}\right) \mathbf{A}_{1} \boldsymbol{\Psi}_{1} \\
\vdots \\
a_{b}^{H}\left(\bar{\theta}_{1}\right) \mathbf{A}_{1} \boldsymbol{\Psi}_{N-2}
\end{array}\right] \boldsymbol{\Psi}_{1} s(t)+n^{\prime \prime}{ }_{2}(t)
\end{gathered}
$$

where $\quad n^{\prime \prime}{ }_{1}(t)=\left[n_{1}^{\prime}(t), n_{2}^{\prime}(t), \ldots, n_{N-1}^{\prime}(t)\right]^{T}, \quad$ and $n^{\prime \prime}{ }_{2}(t)=\left[n_{2}^{\prime}{ }^{T}(t), n_{3}^{\prime}{ }^{T}(t), \ldots, n_{N}^{\prime}{ }^{T}(t)\right]^{T}$.

Clearly, the pair of Equations (14) and (15) has a similar form to that of (4) and (5) and an invariance structure exists between two subarrays. Thus, ESPRIT can be applied to find the estimates of the source DOAs. Note that the noise terms in (14) and (15) is no longer white; the covariance is now $\sigma_{n}^{2} a_{b}^{H}\left(\bar{\theta}_{1}\right) a_{b}\left(\bar{\theta}_{1}\right)$. To get the signal subspace estimate, step 2 in TLSESPRIT needs to be replaced with a generalized eigendecomposition [4].

For conventional ESPRIT processing, to resolve $K$ sources, it is required $N-1 \geq K$. However, beamforming attenuates those sources outside the beam (i.e., the virtual sensor has certain directivity), and usually only source directions within the beam can be estimated. This relaxes the requirement for the size of the virtual array, and $\Psi_{1}$ can be reduced to a small size. On the other hand, beamforming could degrade the performance of the ESPRIT if the beam axis is not enough aligned with the sources. One solution to this problem is to form multiple adjacent beams closelyspaced as discussed in the next section. 
Case 2: $L>1$

For $L>1$, the beamformer output at each subarray is an $L \times 1$ vector:

$$
\begin{gathered}
\mathbf{y}_{1}(t)=\mathbf{W}_{b}^{H} \mathbf{A}_{1} \mathbf{s}(t)+n^{\prime}{ }_{1}(t) \\
\mathbf{y}_{2}(t)=\mathbf{W}_{b}^{H} \mathbf{A}_{1} \boldsymbol{\Psi}_{1} \mathbf{s}(t)+n_{2}^{\prime}(t) \\
\vdots \\
\mathbf{y}_{N}(t)=\mathbf{W}_{b}^{H} \mathbf{A}_{1} \Psi_{N-1} \mathbf{s}(t)+n_{N}^{\prime}(t)
\end{gathered}
$$

where $n_{i}^{\prime}(t)=\mathbf{W}_{b}^{H} \mathbf{n}_{i}(t), i=1, \ldots, N$. Denote $\mathbf{y}(t)=$ $\left[\begin{array}{lll}\mathbf{y}_{1}^{T}(t) & \cdots & \mathbf{y}_{N}^{T}(t)\end{array}\right]^{T}$; then a virtual array of $L \times N$ sensors is formed. We divide it into two subarrays of size $L(N-1)$ denoted by

$$
\begin{aligned}
\mathbf{z}_{1}(t)= & {\left[\begin{array}{c}
\mathbf{W}_{b}^{H} \mathbf{A}_{1} \\
\mathbf{W}_{b}^{H} \mathbf{A}_{1} \boldsymbol{\Psi}_{1} \\
\vdots \\
\mathbf{W}_{b}^{H} \mathbf{A}_{1} \boldsymbol{\Psi}_{N-2}
\end{array}\right] s(t)+n^{\prime \prime}{ }_{1}(t) } \\
\mathbf{z}_{2}(t)= & {\left[\begin{array}{c}
\mathbf{W}_{b}^{H} \mathbf{A}_{1} \\
\mathbf{W}_{b}^{H} \mathbf{A}_{1} \boldsymbol{\Psi}_{1} \\
\vdots \\
\mathbf{W}_{b}^{H} \mathbf{A}_{1} \boldsymbol{\Psi}_{N-2}
\end{array}\right] \boldsymbol{\Psi}_{1} s(t)+n^{\prime \prime}{ }_{2}(t) }
\end{aligned}
$$

where $n^{\prime \prime}{ }_{1}(t)=\left[n_{1}^{\prime}{ }_{1}^{T}(t), n_{2}^{\prime}{ }^{T}(t), \ldots, n_{N-1}^{\prime}{ }^{T}(t)\right]^{T}$, and $n^{\prime \prime}{ }_{2}(t)=\left[n_{2}^{\prime} T(t), n_{3}^{\prime} T(t), \ldots, n_{N}^{\prime}{ }^{T}(t)\right]^{T}$. Again, the pair of Equations (17) and (18) has a similar form to that of (4) and (5), and the invariance structure stays, based on which ESPRIT can be applied. ${ }^{\text {b }}$

Note that a simple choice of $L=2$ or 3 is often enough to improve the beam-domain DOA estimation performance. Hence, the dimension in the beam-domain can still be far less than that in the element space, reducing the computational complexity and the number of snapshots needed for statistically robust covariance estimation. Specifically, for the same number of subarrays $N$ and the same number of snapshots $Q$, the computation time of the original ESPRIT is on the order of the maximum of $P^{3}$ and $Q P^{2}$, while that of ESPRIT with subarray beamforming is on the order of the maximum of $Q L P$, $Q L^{2}$, and $L^{3}$. The extra computation of the subarray beamforming is minor, especially when $L \ll P$. Moreover, forming $L$ different beam pointing angles can be realized in parallel, and $Q$ can be much smaller for the subarray-beamforming approach. Hence, for $L \ll P$, ESPRIT with subarray beamforming exhibits significant computational advantages as compared to the original ESPRIT method.

In practical multi-beam system applications, the above method can be applied to the selected beams following conventional beamforming generating beams at a set of pre-selected angles and the subsequent source signal detection at each beam.

\section{Simulations}

To assess the performance of ESPRIT with subarray beamforming (we denote it as ESPRIT-SB), we look at a number of examples of DOA estimation using the TLS version of both ESPRIT and ESPRIT-SB. A 64-sensor uniform linear array with sensor spacing equal to ${ }^{7} \lambda / 16$ is configured, thus the $3 \mathrm{~dB}$ beamwidth of the effective aperture near broadside is about $1.83^{\circ}$. The number of sources is assumed to be known, and individual source processes are uncorrelated and corrupted by white Gaussian noises. The result presented is the individual or averaged root-mean-squared-errors (RMSE) in estimation of all the source DOA's, evaluated from 1500 MonteCarlo runs.

Consider first that two equal-power incident signals come from $\theta_{1}=25.6^{\circ}$ and $\theta_{2}=26.5^{\circ}$, respectively, which are separated by less than one beamwidth. Using conventional beamforming, 225 beams are formed uniformly spanning $-90^{\circ}$ to $90^{\circ}$, and the output result is given in Figure 1. It is clearly shown that the beam covering an angular range of $\left[24.9^{\circ}, 26.5^{\circ}\right]$ embodies those two sources. To implement ESPRIT-SB, the angles of the subarray beams can arbitrarily be chosen as long as they cover one or a few consecutive conventional beams. In the following simulations, they are determined from

$$
\bar{\theta}_{l}=24.9^{\circ}+\frac{\left(26.5^{\circ}-24.9^{\circ}\right) l}{L+1}, l=1, \ldots, L
$$

We divide the array into $N=4$ subarrays, each with 49 sensors and equally separated by $5 d$. Figure 2 illustrates the average DOA estimation performance as a function of the number of selected beams at different SNR conditions. The number of snapshots used is 20. Compared

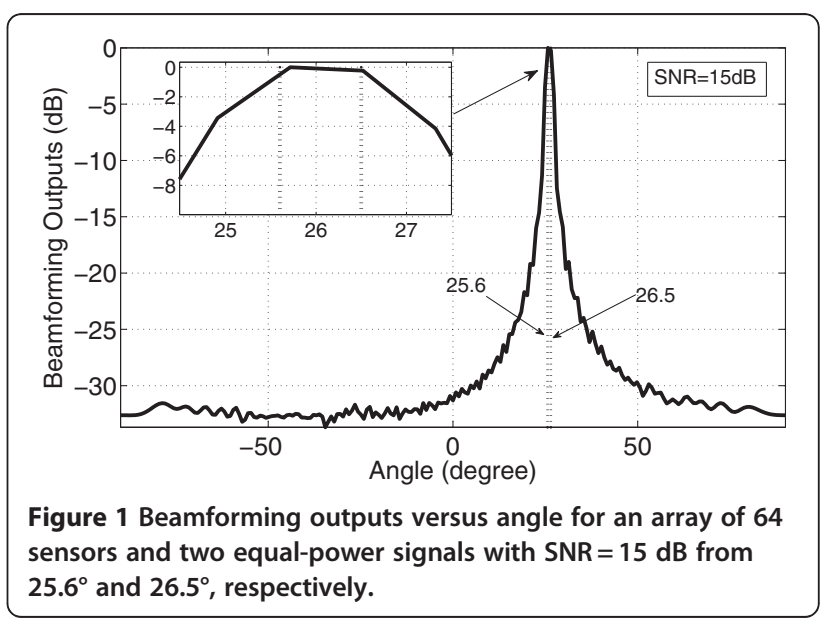




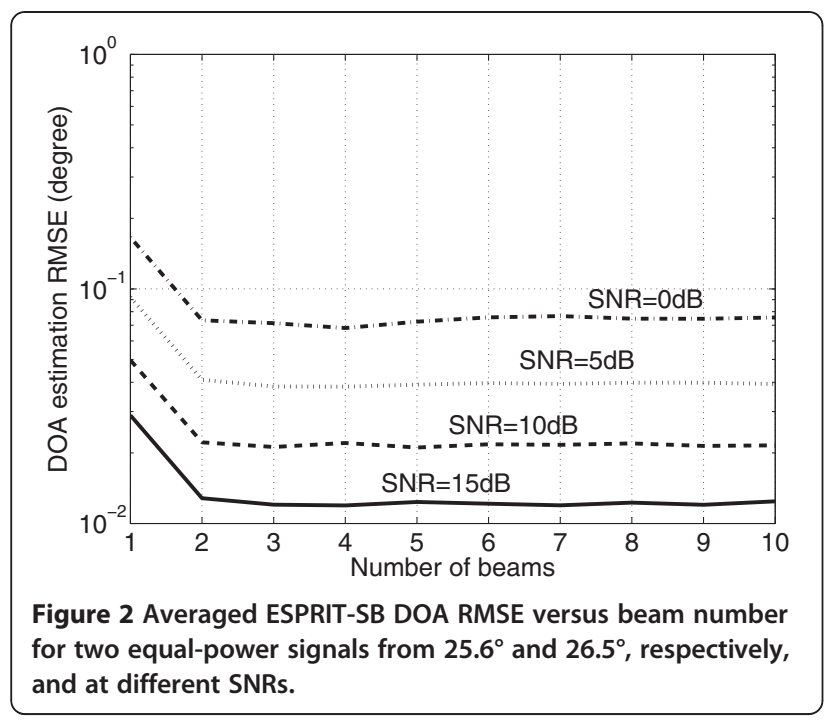

to the single-beam case, the performance improves dramatically when one additional beam is added, demonstrating the effectiveness of the approach. On the other hand the RMSE is almost a constant for $L \geq 2$, which is highly desired as discussed in the last section.

Figure 3 displays the results of dividing the array into different number of subarrays with different separations. For Figure $3 \mathrm{a}$, the subarray spacing is fixed at $5 d$; for Figure $3 \mathrm{~b}$, the subarray number is fixed at 4 . RMSE of the ESPRIT-SB with $L=1$ and $L=2$ (denoted by ESPRIT-SB-1 and ESPRIT-SB-2, respectively) is evaluated. The SNR is $-5 \mathrm{~dB}$ and 50 snapshots are used to match the maximum subarray number in this example. Note that the way the subarrays are formed here causes a tradeoff in SNR gain, i.e., increasing the subarray number (thus the ESPRIT processing gain) means a

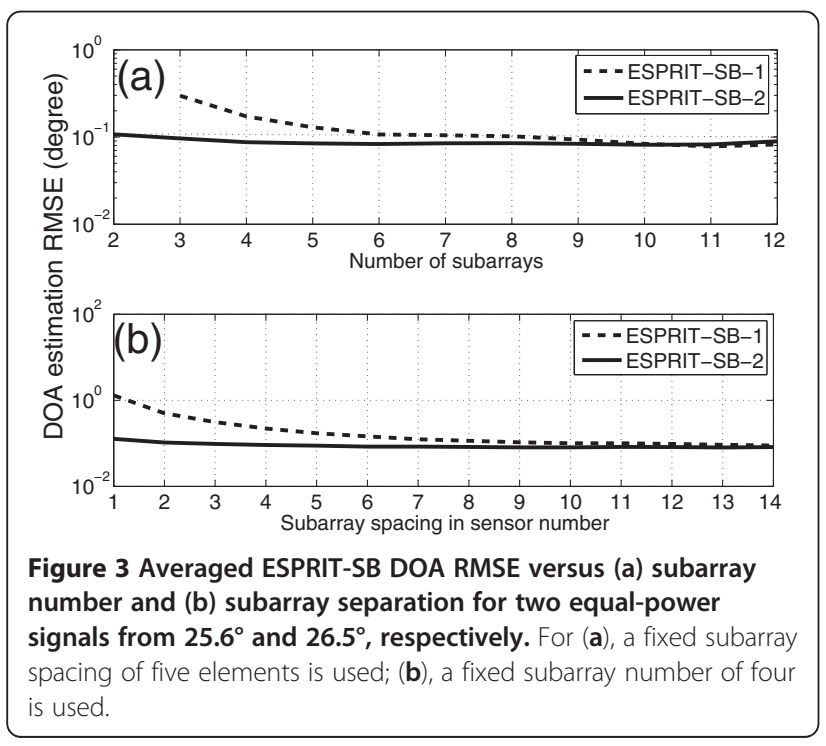

reduction of subarray length (thus the beamforming processing gain). Besides, different subarray overlapping introduces different respective signal and noise correlations, which affects the SNR gain as well.

Behaviors in Figure 3 can be understood as a combined effect of the above-mentioned factors. While performance of the ESPRIT-SB-1 improves as the number of subarrays or subarray spacing increases (and thus a wider beamwidth), ESPRIT-SB-2 performs uniformly better and does not show much variation over the chosen variable intervals. The results here along with that in Figure 2 show that ESPRIT-SB with only one formed beam is quite sensitive to scalloping loss in beamforming. It is interesting to also note that resolving two sources is realized by two subarrays (virtual sensors), thank to one additional beam formed at each subarray.

To further demonstrate the above observations, two more examples are considered. The first one assumes that two equal-power incident signals arrive from $\theta_{1}=$ $55.6^{\circ}$ and $\theta_{2}=56.1^{\circ}$, respectively. Figures 4 and 5 present the results similar to Figures 2 and 3, except the $\mathrm{SNR}$ is $5 \mathrm{~dB}$ in Figure 5 while $-5 \mathrm{~dB}$ in Figure 3. The second example considers that three equal-power incident signals arrive from $\theta_{1}=25.6^{\circ}, \theta_{2}=26.3^{\circ}$, and $\theta_{3}=27.0^{\circ}$, respectively. Figures 6 and 7 show the results similar to Figures 2 and 3, except the SNR is $5 \mathrm{~dB}$ in Figure 7 also. Clearly, some similar behaviors mentioned above can be seen in those figures, respectively.

As the last set of examples, we compare ESPRIT-SB to the conventional ESPRIT implemented on the entire (real) array, i.e., without subarray beamforming. For two sources and ESPRIT-SB, $N=4, \Delta=5 d$, and $P=49$; for the conventional ESPRIT, $M=64$ and $M_{s}=63$. For both algorithms, 200 snapshots are used. To further compare with some theoretical performance analysis

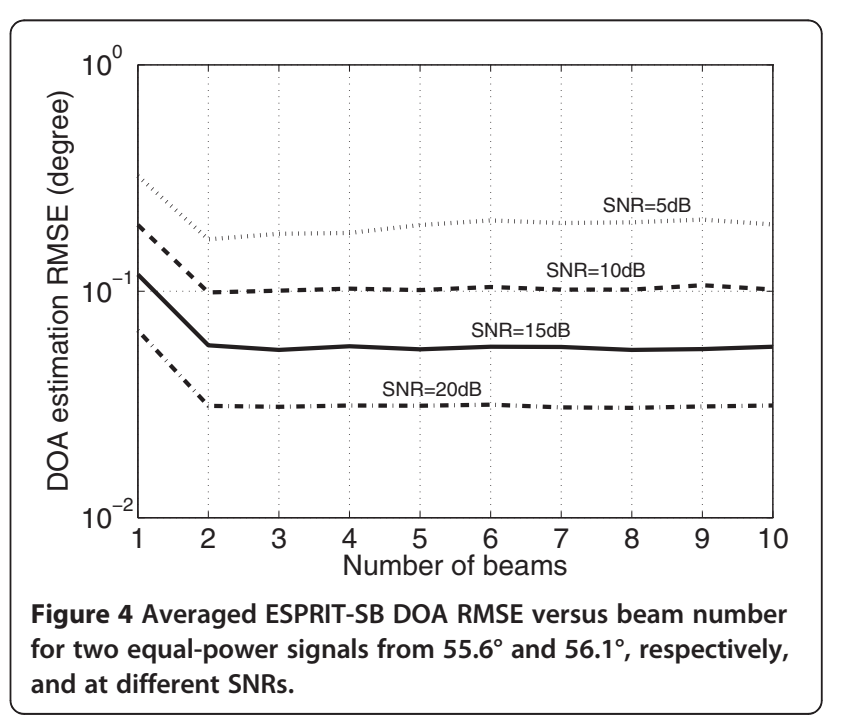




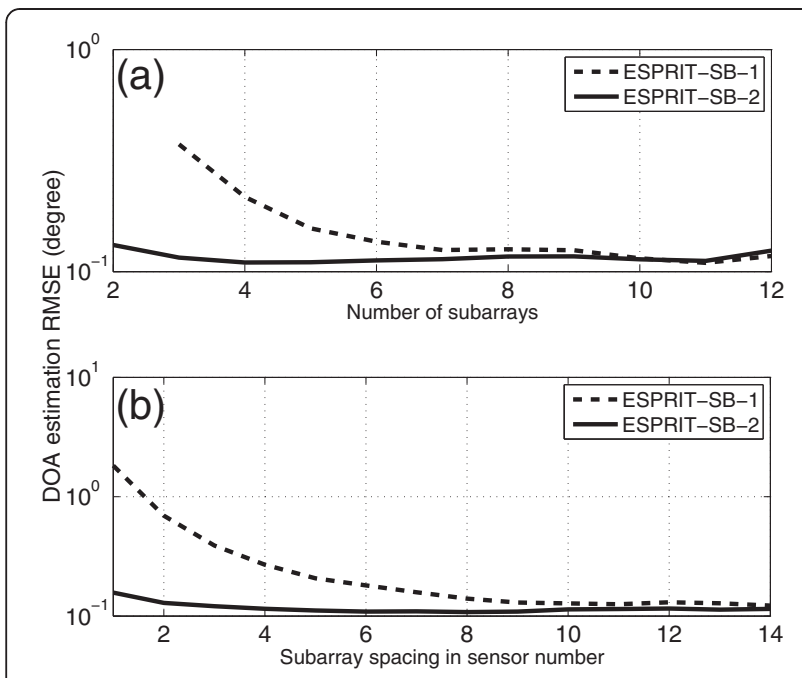

Figure 5 Averaged ESPRIT-SB DOA RMSE versus (a) subarray number and (b) subarray separation for two equal-power signals from $55.6^{\circ}$ and $56.1^{\circ}$, respectively. For (a), a fixed subarray spacing of five elements is used; (b), a fixed subarray number of four is used. The SNR is $5 \mathrm{~dB}$ and 50 snapshots are used

results, the Cramer-Rao bound for the same problem setup is presented, which is evaluated from [13]:

$$
\mathrm{CRB}(\boldsymbol{\theta})=\frac{\sigma_{n}^{2}}{2 Q}\left\{\operatorname{Re}\left[\left(\mathbf{D}^{H} \mathbf{P}_{\mathbf{A}}^{\perp} \mathbf{D}\right) \odot\left(\mathbf{R}_{\mathbf{s s}} \mathbf{A}^{H} \mathbf{R}_{\mathbf{x x}}^{-1} \mathbf{A} \mathbf{R}_{\mathbf{s s}}\right)^{T}\right]\right\}^{-1}
$$

where $\quad \mathbf{D}=\left[\begin{array}{lll}\mathbf{d}_{1} & \cdots & \mathbf{d}_{K}\end{array}\right] ; \mathbf{d}_{k}=d \mathbf{a}\left(\theta_{k}\right) / d \theta_{k}, \mathbf{P}_{\mathbf{A}}^{\perp}=$ $\mathbf{I}_{M}-\mathbf{P}_{\mathbf{A}} ; \mathbf{P}_{\mathbf{A}}=\mathbf{A}\left(\mathbf{A}^{H} \mathbf{A}\right)^{-1} \mathbf{A}^{H}$, and $\odot$ denotes the Hadamard-Schur product.

The results of RMSE for each source are plotted as a function of SNR in Figures 8 and 9, respectively. Note that, the SNR approaches $-30 \mathrm{~dB}$, the RMSE approaches

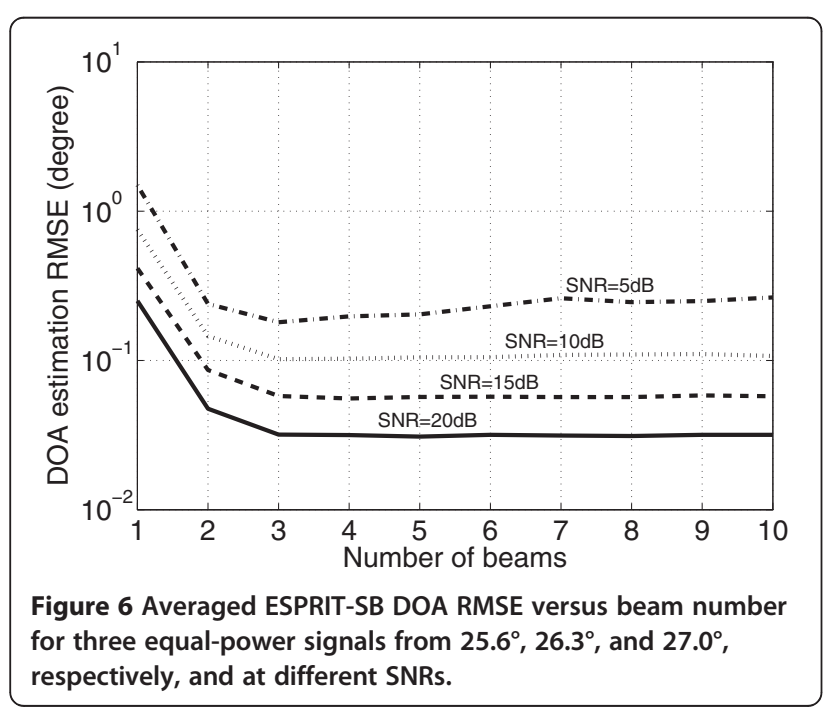

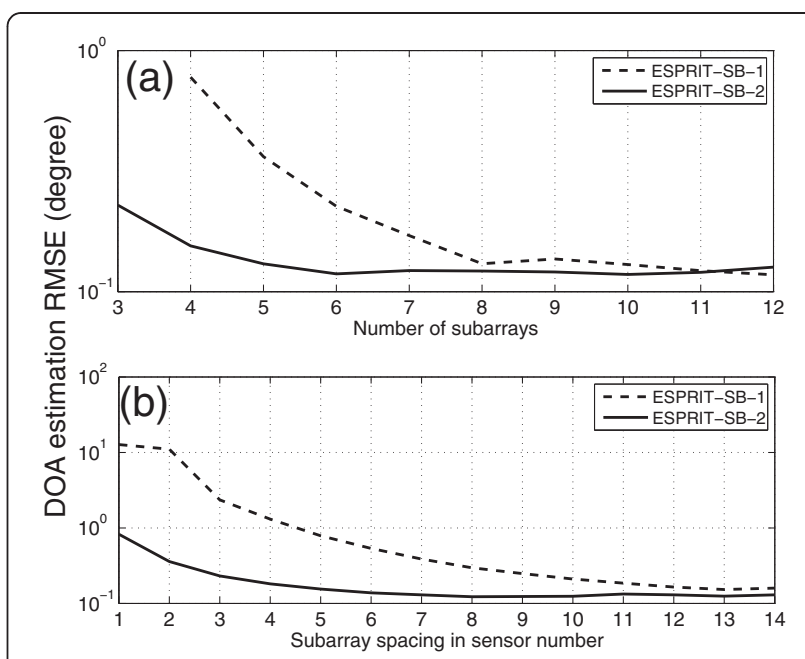

Figure 7 Averaged ESPRIT-SB DOA RMSE versus (a) subarray number and (b) subarray separation for three equal-power signals from $25.6^{\circ}, 26.3^{\circ}$, and $27.0^{\circ}$, respectively. For (a), a fixed subarray spacing of five elements is used; (b), a fixed subarray number of four is used. The SNR is $5 \mathrm{~dB}$ and 50 snapshots are used

the variance of a random variable that is uniform [14]. As expected, the RMSE presents a threshold behavior, that is, below some SNR, RMSE increases dramatically due to ambiguity sidelobes [14]. An SNR gain on the order of $10 \log P$ can clearly be seen: the element-space ESPRIT enters the threshold region earlier, and ESPRITSB-1 performs the same with the element-space ESPRIT in the high-SNR region, while ESPRIT-SB-2 always performs better there.

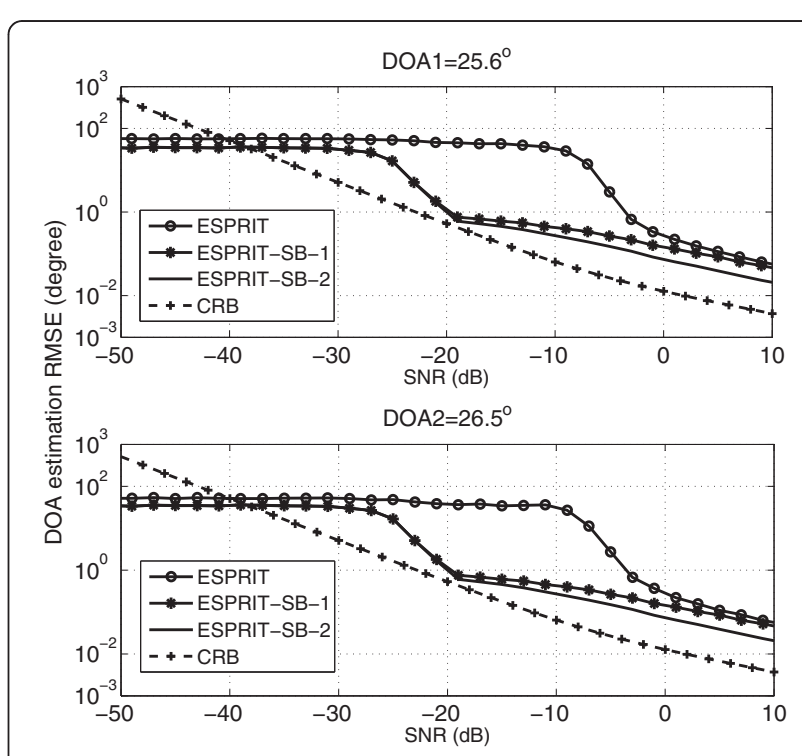

Figure 8 ESPRIT-SB DOA RMSE versus SNR for two equal-power signals from $25.6^{\circ}$ and $26.5^{\circ}$, respectively, with a fixed subarray spacing of five elements and subarray number of four. The element-space ESPRIT result and CRB are also shown. 


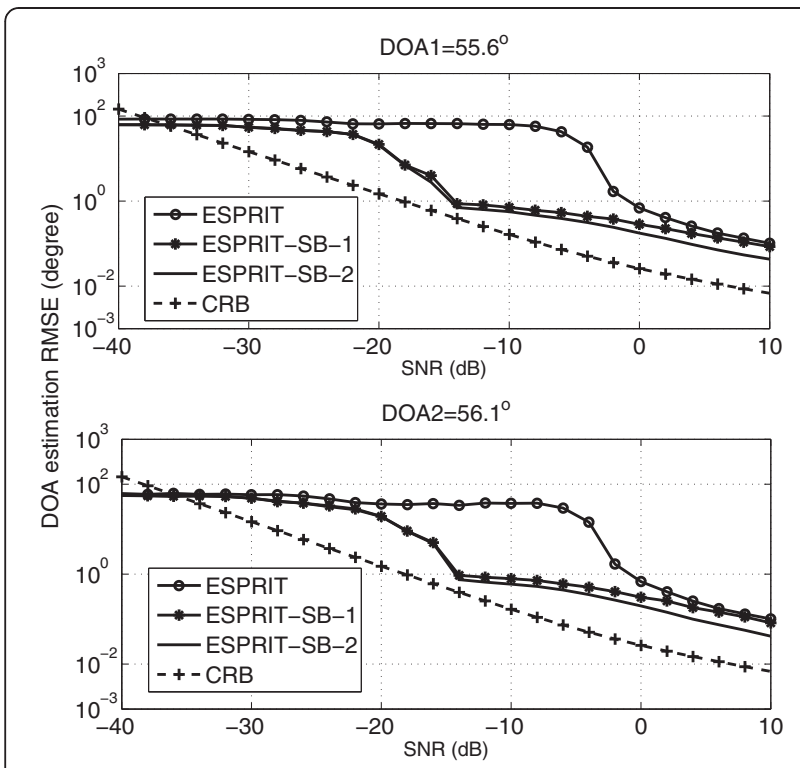

Figure 9 ESPRIT-SB DOA RMSE versus SNR for two equal-power signals from $55.6^{\circ}$ and $56.1^{\circ}$, respectively, with a fixed subarray spacing of five elements and subarray number of four. The element-space ESPRIT result and CRB are also shown

It is interesting to note that above threshold, the CRB is always lower than the simulated RMSE. Indeed when the number of sensors gets down, say 10, the gap would be much smaller, e.g., $1 \mathrm{~dB}$. A similar observation can be found in [14, p. 1193]. It could be that as the subarray size increases, subarray overlapping ratio increases resulted in increased respective signal and noise correlations, which is not considered in the CRB.

\section{Conclusion}

An ESPRIT-based DOA estimation algorithm with multi-angle subarray beamforming is presented. As a beam-domain method, it works for relatively smaller number of snapshots and lower SNR and may render better numerical stability in computation, compared to the conventional element-space ESPRIT. Simulations show that with a few more closelyspaced beams formed, capability of resolving closelyspaced sources within a conventional beam is greatly enhanced. The developed approach is particularly useful in multi-beam system applications where a sub-beamwidth resolution is desired with a limited number of data snapshots, for example in bottom detection processing of a multi-beam echo sounder.

\section{Methods}

All of the signals used in this article are produced by Matlab software, and the algorithms in the simulation section are based on the results proposed in Section "Problem statement" and Section "ESPRIT applied to multiple-angle subarray beams".

\section{Endnotes}

${ }^{a}$ To distinguish from subarray structuring in ESPRIT, here we use a different set of notations.

${ }^{\mathrm{b}}$ An alternative derivation may be done in the context of vector-sensor ESPRIT [15], treating each subarraybeamformer output as a virtual vector sensor, which is though beyond the scope of this article.

\section{Competing interests}

The authors declare that they have no competing interests.

\section{Acknowledgments}

The authors wish to thank all the anonymous reviewers for their valuable comments. This study was supported by the Chinese 863 high-tech program under Grant 2007AA09Z125 and Chinese NSF under Grant 60872067.

Received: 1 June 2011 Accepted: 10 June 2012

Published: 20 July 2012

\section{References}

1. C de Moustier, State of the art in swath bathymetry survey systems. Int. Hydrographic Rev. 65(2), 25-54 (1988)

2. R Roy, T Kailath, ESPRIT: estimation of signal parameters via rotational invariance techniques. IEEE Trans. Acoust. Speech Signal Process ASSP-37. 984-995 (1989)

3. XL Xu, K Buckley, Reduced-dimension beamspace broadband source localization: preprocessor design and evaluation, in Proc. IEEE ASSP 4th Workshop Spectrum Estimation Modeling (, Minneapolis, MN, ), pp. 22-27. 3-5 Aug 1988

4. J Li, Improving ESPRIT via beamforming. IEEE Trans. Aerospace Electron. Syst. 28(2), 520-527 (1992)

5. G Xu, SD Silverstein, RH Roy, T Kailath, Beamspace ESPRIT. IEEE Trans Signal Process 42(2), 349-356 (1994)

6. H Li, B Yao, T Zhou, Y Wei, B Chen, X Liu, H Yu, N Weng, Proc. of MTS/IEEE OCEANS Conference, in Shallow water high resolution multi-beam echo sounder (, 2008), pp. 186-191

7. AL Swindlehurst, B Ottersten, R Roy, T Kailath, Multiple invariance ESPRIT. IEEE Trans Signal Process 40(4), 867-881 (1992)

8. KT Wong, MD Zoltowski, Closed-form multi-dimensional multi-invariance ESPRIT. Proceedings of ICASSP-97 vol. 5, 3489-3492 (1997)

9. $Y$ Xu, Z Liu, Closed-form multiple invariance ESPRIT. Multidimed.Syst. Signal Process 18(1), 47-54 (2007)

10. A Richter, RS Thoma, Angle estimation with circular uniform beam arrays using the CUBA ESPRIT. Millennium Conference on Antennas and Propagation AP (2000)

11. E Taillefer, A Hirata, T Ohira, Reactance-domain ESPRIT algorithm for a hexagonally shaped seven-element ESPAR antenna. IEEE Trans. Antennas Propag. 53(11), 3486-3495 (2005)

12. E Taillefer, W Nomura, J Cheng, M Taromaru, Y Watanabe, T Ohira, Enhanced reactance-domain ESPRIT algorithm employing multiple beams and translational-invariance soft selection for Direction-of-Arrival estimation in the full azimuth. IEEE Trans. Antennas Propag. 56(8), 2514-2526 (2008)

13. P Stoica, EG Larsson, AB Gershman, The stochastic CRB for array processing: a textbook derivation. IEEE Signal Process. Lett. 8(5), 148-150 (2001)

14. HL Van Trees, Optimum Array Processing (Wiley, New York, 2002)

15. KT Wong, MD Zoltowski, Uni-vector-sensor ESPRIT for multisource azimuth, elevation, and polarization estimation. IEEE Trans. Antennas Propag. 45(10), 1467-1474 (1997)

doi:10.1186/1687-6180-2012-152

Cite this article as: Xu et al:: ESPRIT with multiple-angle subarray beamforming. EURASIP Journal on Advances in Signal Processing 2012 2012:152. 